\title{
A Sharp Divergence Theorem with Nontangential Traces
}

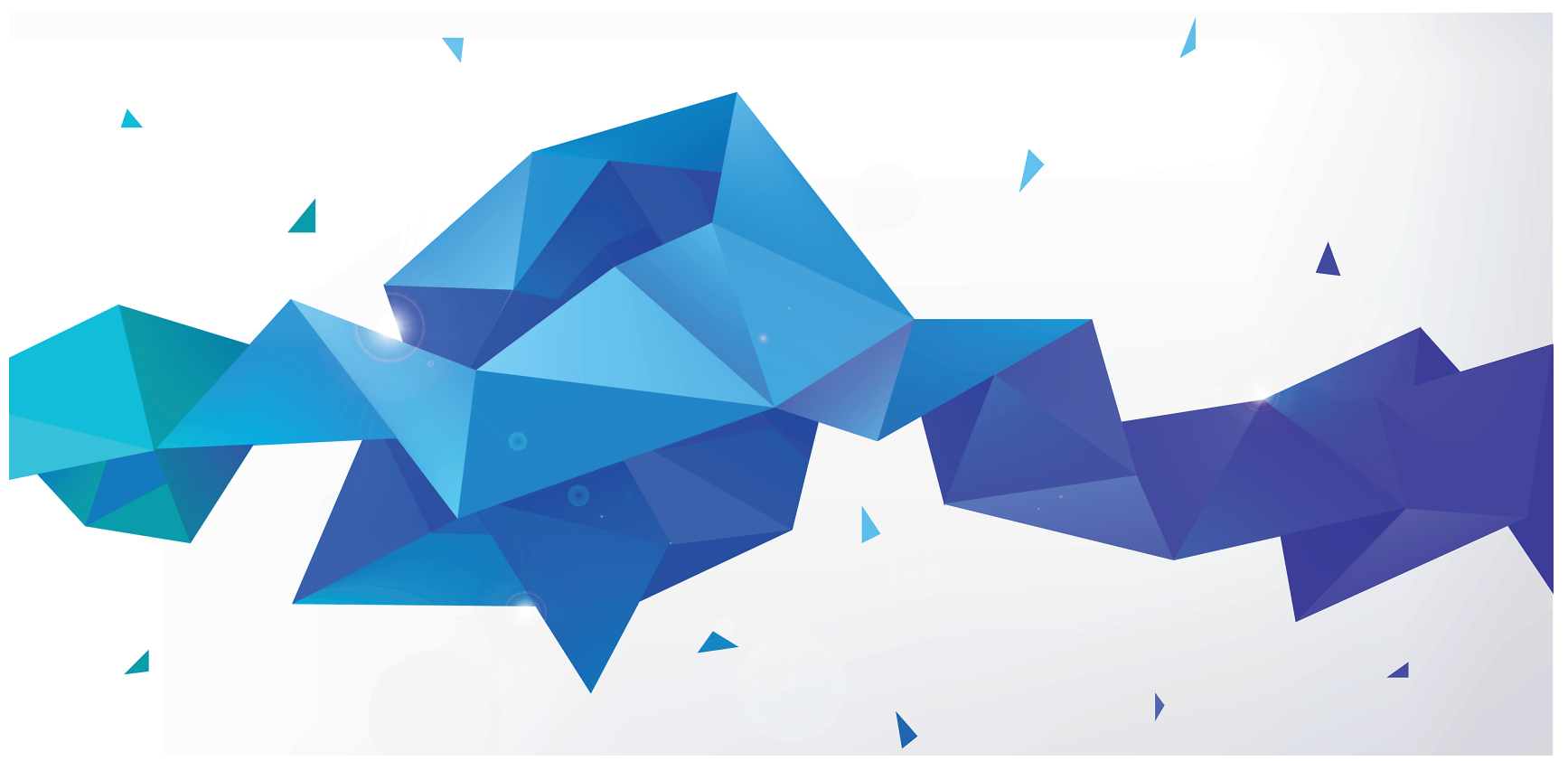

Dorina Mitrea, Irina Mitrea, and Marius Mitrea

\section{A Brief Historical Perspective}

The Fundamental Theorem of Calculus, one of the most spectacular scientific achievements, stands as beautiful, powerful, and relevant today as it did more than three centuries ago when it first emerged onto the mathematical scene. Typically, Isaac Newton and Gottfried Leibniz are credited with developing much of the mathematical machinery associated with this result into a coherent theory for infinitesimal quantities (the bedrock of modern calculus), a mathematical landscape within which the Fundamental Theorem of Calculus stands out as the crowning achievement. In its sharp one-dimensional version, involving the class $\mathrm{AC}([a, b])$ of absolutely continuous

Dorina Mitrea is a professor of mathematics at Baylor University. Her email address is Dorina_Mitrea@baylor.edu.

Irina Mitrea is a professor of mathematics at Temple University. Her email address is imitrea@temple.edu.

Marius Mitrea is a professor of mathematics at Baylor University. His email address is Marius_Mi trea@baylor.edu.

Communicated by Notices Associate Editor Daniela De Silva.

For permission to reprint this article, please contact:

reprint-permission@ams . org.

DOI: https://doi.org/10.1090/noti2149 functions on a compact interval $[a, b]$, the Fundamental Theorem of Calculus reads:

$$
\begin{aligned}
& \int_{a}^{b} F^{\prime}(x) d x=F(b)-F(a) \\
& \text { for every } F \in \operatorname{AC}([a, b]) .
\end{aligned}
$$

It is a stark example of how local information, encoded in the instantaneous rate of change (aka derivative) $F^{\prime}$, can be pieced together via integration to derive conclusions of a global nature about the variation of $F$ over $[a, b]$, a fundamental paradigm in calculus.

Intriguingly, while (1.1) is essentially optimal, dealing with higher-dimensional versions of the Fundamental Theorem of Calculus remains an active area of research in contemporary mathematics. In its standard version, with $\mathcal{L}^{n}$ denoting the $n$-dimensional Lebesgue measure in $\mathbb{R}^{n}$, the Divergence Theorem asserts that

if $\Omega \subseteq \mathbb{R}^{n}$ is a bounded domain of class $\mathcal{C}^{1}$, with outward unit normal $\nu$ and surface measure $\sigma$, then $\int_{\Omega} \operatorname{div} \vec{F} d \mathcal{L}^{n}=\int_{\partial \Omega} \nu \cdot\left(\left.\vec{F}\right|_{\partial \Omega}\right) d \sigma$ for each vector field $\vec{F} \in\left[\mathcal{C}^{1}(\bar{\Omega})\right]^{n}$. 
Since the divergence of a continuously differentiable vector field $\vec{F}$ may be computed pointwise as $(\operatorname{div} \vec{F})(x)=$ $\lim _{r \rightarrow 0^{+}} \mathcal{L}^{n}(B(x, r))^{-1} \int_{\partial B(x, r)}\left(\frac{y-x}{r}\right) \cdot \vec{F}(y) d \mathcal{H}^{n-1}(y)$, where $\mathcal{H}^{n-1}$ stands for the $(n-1)$-dimensional Hausdorff measure in $\mathbb{R}^{n}$, it follows that the quantity $(\operatorname{div} \vec{F})(x)$ is emblematic of the tendency of a vector field $\vec{F}$ to collect (sink effect) or disperse (source effect) at a point $x$. In view of this feature, the Divergence Formula in (1.2) may be regarded as a conservation law, asserting that the solid integral of all such sources and sinks associated with a given vector field is equal to the net flow of said vector field through the solid's boundary. ${ }^{1}$ Hence, in complete analogy to the Fundamental Theorem of Calculus mentioned earlier, the Divergence Theorem describes how the infinitesimal sink/source effects created by a vector field may be pieced together inside a given domain to produce a global, macroscopic effect along the boundary.

The classical result recorded in (1.2) is usually associated with the names of J.-L. Lagrange, who first established a special case of the Divergence Theorem in 1762 working on the propagation of sound waves (cf. [14]); C. F. Gauss, who independently considered a particular case in 1813 (cf. [9]); M. V. Ostrogradsky, who gave the first proof of the general theorem in 1826 (cf. [20]); G. Green, who used a related formula in 1828 (cf. [10]); A. Cauchy, who in 1846 first published, without proof, the nowadays familiar form of Green's theorem (cf. [1]); B. Riemann, who provided a proof of Green's formula in his 1851 inaugural dissertation (see [21]); Lord Kelvin, who in 1850 discovered a special version of Stokes' theorem (in the three-dimensional setting, also known as the curl theorem); and É. Cartan, who first published the general form of Stokes' theorem (in the language of differential forms on manifolds) in 1945, among others. However, a precise attribution is fraught with difficulty since the Divergence Theorem in its modern format has undergone successive waves of reformulations and generalizations, as well as more rigorous proofs, with inputs from a multitude of sources (general historical accounts may be found in [12] and [22]).

Specializing the Divergence Formula in (1.2) to the case when $\vec{F}$ is the restriction to $\bar{\Omega}$ of vector fields from $\left[\mathcal{C}_{c}^{\infty}\left(\mathbb{R}^{n}\right)\right]^{n}$ (where $\mathcal{C}_{c}^{\infty}\left(\mathbb{R}^{n}\right)$ denotes the space of smooth, compactly supported functions in $\mathbb{R}^{n}$ ) yields the statement:

if $\Omega \subseteq \mathbb{R}^{n}$ is a bounded domain of class $\mathcal{C}^{1}$, with outward unit normal $\nu$ and surface measure $\sigma$, then $\nabla \mathbf{1}_{\Omega}=-\nu \sigma$ in $\left[\mathcal{D}^{\prime}\left(\mathbb{R}^{n}\right)\right]^{n}$,

\footnotetext{
${ }^{1}$ For instance, imagining $\vec{F}$ as the velocity field of an incompressible fluid flow occupying a fixed region $\Omega$, this informally states that "what goes in must come out."
}

where $\mathbf{1}_{\Omega}$ is the characteristic function of $\Omega$ and $\mathcal{D}^{\prime}\left(\mathbb{R}^{n}\right)$ denotes the space of distributions in $\mathbb{R}^{n}$. Conversely, since both $\nabla \mathbf{1}_{\Omega}$ and $-\nu \sigma$ are vector distributions in $\mathbb{R}^{n}$ of order $\leq 1$, their actions canonically extend to vector fields from $\left[\mathcal{C}_{c}^{1}\left(\mathbb{R}^{n}\right)\right]^{n}$, in which scenario we recover (1.2). Hence, (1.3) amounts to an equivalent reformulation of the classical Divergence Theorem (1.2), which has a purely geometric measure theoretic nature. In particular, (1.3) brings into focus the fact that the distributional gradient of the characteristic function of a bounded $\mathcal{C}^{1}$ domain is a locally finite Borel vector-valued measure in $\mathbb{R}^{n}$.

As far as the latter property is concerned, R. Caccioppoli, E. De Giorgi, and H. Federer registered a decisive leap forward by considering the largest class of Euclidean subsets enjoying said property, i.e., the class of sets of locally finite perimeter. It turns out that this consists of Lebesgue measurable subsets $\Omega$ of $\mathbb{R}^{n}$ with the property that $\mathbf{1}_{\Omega}$ is of locally bounded variation in $\mathbb{R}^{n}$, i.e., $\mathbf{1}_{\Omega} \in \mathrm{BV}_{\text {loc }}\left(\mathbb{R}^{n}\right)$. In turn, membership to $\mathrm{BV}_{\text {loc }}\left(\mathbb{R}^{n}\right)$ is conceived in such a way that the Riesz Representation Theorem can naturally be applied to the functional

$$
\Lambda(\vec{F}):=\int_{\Omega} \operatorname{div} \vec{F} d \mathcal{L}^{n} \text { for all } \vec{F} \in\left[\mathcal{C}_{c}^{1}\left(\mathbb{R}^{n}\right)\right]^{n},
$$

to conclude that there exist a Borel measure $\sigma_{*}$ in $\mathbb{R}^{n}$, which is actually supported on $\partial \Omega$, and a $\sigma_{*}$-measurable vector-valued function $\nu: \mathbb{R}^{n} \rightarrow \mathbb{R}^{n}$ satisfying $|\nu|=1$ at $\sigma_{*}$-a.e. point in $\mathbb{R}^{n}$, such that

$$
\Lambda(\vec{F})=\int_{\mathbb{R}^{n}} \nu \cdot \vec{F} d \sigma_{*} \text { for all } \vec{F} \in\left[\mathcal{C}_{c}^{1}\left(\mathbb{R}^{n}\right)\right]^{n} .
$$

The function $v$ is referred to as the geometric measure theoretic outward unit normal to $\Omega$. Bearing in mind that $\sigma_{*}$ is actually supported on $\partial \Omega$, the following version of the Divergence Theorem emerges from (1.4) and (1.5):

$$
\begin{aligned}
& \int_{\Omega} \operatorname{div} \vec{F} d \mathcal{L}^{n}=\int_{\partial \Omega} \nu \cdot \vec{F} d \sigma_{*} \\
& \text { for each vector field } \vec{F} \in\left[\mathcal{C}_{c}^{1}\left(\mathbb{R}^{n}\right)\right]^{n} .
\end{aligned}
$$

The real achievement of De Giorgi and Federer is further refining (1.6) by establishing that actually

$$
\sigma_{*}=\mathcal{H}^{n-1}\left\lfloor\partial_{*} \Omega,\right.
$$

where $\partial_{*} \Omega$ denotes the measure theoretic boundary of $\Omega$, defined as

$$
\begin{array}{r}
\partial_{*} \Omega:=\left\{x \in \mathbb{R}^{n}: \limsup _{r \rightarrow 0^{+}} \frac{\mathcal{L}^{n}(B(x, r) \cap \Omega)}{r^{n}>0}\right. \text { and } \\
\left.\limsup _{r \rightarrow 0^{+}} \frac{\mathcal{L}^{n}(B(x, r) \backslash \Omega)}{r^{n}}>0\right\} .
\end{array}
$$

Hence, near points in $\partial_{*} \Omega$ there is enough mass (relative to the scale) both in $\Omega$ and in $\mathbb{R}^{n} \backslash \Omega$. Let us also note that, in 
principle, $\partial_{*} \Omega$ can be a much smaller set than the topological boundary $\partial \Omega$. Substituting (1.7) back into (1.6) then yields the following result:

Theorem 1.1 (De Giorgi-Federer's Divergence Theorem [4, $5,7,8])$. Let $\Omega \subseteq \mathbb{R}^{n}$ be a set of locally finite perimeter. Let $\nu$ be the geometric measure theoretic outward unit normal to $\Omega$, and let $\sigma:=\mathcal{H}^{n-1}\left[\partial \Omega\right.$. Then for each vector field $\vec{F} \in\left[\mathcal{C}_{\mathcal{c}}^{1}\left(\mathbb{R}^{n}\right)\right]^{n}$ one has

$$
\left.\int_{\Omega}(\operatorname{div} \vec{F})\right|_{\Omega} d \mathcal{L}^{n}=\int_{\partial_{*} \Omega} \nu \cdot\left(\left.\vec{F}\right|_{\partial_{*} \Omega}\right) d \sigma .
$$

In a nutshell, one of the key results of the De GiorgiFederer theory is the identity

$$
\nabla \mathbf{1}_{\Omega}=-v \mathcal{H}^{n-1}\left\lfloor\partial_{*} \Omega\right.
$$

as distributions in $\mathbb{R}^{n}$, whenever

$\Omega \subseteq \mathbb{R}^{n}$ is a set of locally finite perimeter,

which may then readily be reinterpreted as the Divergence Formula (1.8) simply by untangling jargon. A timely exposition may be found in $[6, \$ 5.8$, Theorem 1, p. 209]. For the original work see [4], [5], [7], [8], as well as [2] for additional comments and references.

The nature of the Divergence Theorem is such that the smoother the category of vector fields considered, the rougher the class of domains which may be allowed in the formulation of said theorem. While the De Giorgi-Federer version of the Divergence Theorem applies to a large class of domains (i.e., sets of locally finite perimeter), the vector fields involved are assumed to have components in $\mathcal{C}_{c}^{1}\left(\mathbb{R}^{n}\right)$. Thus, the vector fields in the De Giorgi-Federer version of the Divergence Theorem belong to a very restrictive class, are exceedingly regular, as well as completely unrelated to the underlying domain. Moreover, when specialized to the case $n=1$, for a finite interval of the real line, the De Giorgi-Federer version of the Divergence Theorem formulated in Theorem 1.1 fails to yield the sharp version of the Fundamental Theorem of Calculus, recorded in (1.1).

While formula (1.8) has been successfully used in many branches of mathematics, Theorem 1.1 is not adequate for a variety of problems in partial differential equations, scattering, and harmonic analysis, since in many fundamental instances $\vec{F}$ is not continuous up to and including the boundary, but rather the trace of $\vec{F}$ to $\partial \Omega$ is considered in a pointwise nontangential sense. As such, one needs a divergence formula for rough integrands and rough boundaries that can handle these cases. Of course, any significant weakening of the assumptions on the vector field $\vec{F}$ in Theorem 1.1 should be accompanied by a corresponding strengthening of the assumptions on the underlying domain $\Omega$. Ad hoc techniques, based on approximating the original set $\Omega$ by a suitable sequence of subdomains $\Omega_{j} \nearrow \Omega$, have sufficed for continuous vector fields in
Lipschitz domains, and also for a class of Reifenberg-flat domains (cf. [3], [13]), but to go beyond this one needs genuinely new techniques. Progress in this regard has been registered in [11], which treats a much larger class of domains than Lipschitz, without any flatness assumptions. However, the version of the Divergence Theorem established in [11] requires that the (nontangential) trace of the vector field $\vec{F}$ on the boundary is $p$ th power integrable for some $p>1$. This requirement is an artifact of the proof, which relies on the boundedness of the Hardy-Littlewood maximal function on $L^{p}$ with $p>1$.

The goal of this article is to describe a new brand of Divergence Theorem developed in [17] exhibiting the following features (all of which are absent from De GiorgiFederer's version of the Divergence Theorem recorded in Theorem 1.1):

- when $n=1$ and $\Omega$ is a finite interval on the real line, our theorem reduces precisely to the sharp version of the Fundamental Theorem of Calculus formulated in (1.1);

- the vector field $\vec{F}$ is intrinsically defined in $\Omega$, and may lack continuity, or even local boundedness;

- the divergence of $\vec{F}$ is computed in the sense of distributions and is allowed to exhibit certain types of singularities;

- the only quantitative aspect not directly associated with the ability of writing the two integrals making up the Divergence Formula in a meaningful way is an integrability condition imposed on the nontangential maximal function of the vector field $\vec{F}$;

- the trace of $\vec{F}$ on the boundary is considered in a pointwise nontangential sense (i.e., considering the limit of $\vec{F}$ from within certain nontangential approach regions with vertices at points on $\partial \Omega$ ).

Compared with the classical results of De GiorgiFederer, our work brings into focus the role of the nontangential maximal operator and the nontangential boundary trace in the context of the Divergence Theorem. The idea of imposing an integrability condition on the nontangential maximal operator and then using this to prove the existence of nontangential boundary limits originates in the classical work of Fatou. In particular, the class of functions for which such a nontangential boundary trace exists serves as a natural enlargement of the category of functions which are continuous up to, and including, the topological boundary of the underlying domain. In a broader perspective, describing the qualitative and quantitative boundary behavior of a function via its nontangential boundary trace and its nontangential maximal operator is a natural point of view which has been adopted in a multitude of branches of analysis. Concrete examples of this flavor, highlighting the adequacy and appropriateness of taking boundary traces in a nontangential pointwise sense, and 
imposing integrability conditions on the nontangential maximal operator, include: Hardy space of holomorphic functions, singular integral operators of CalderónZygmund-type, boundary value problems in rough domains, and Fatou-type results.

\section{Divergence Theorems with Nontangential Pointwise Traces}

Our first main result pertains to the Divergence Theorem in its standard format, as the equality between the solid integral of the divergence of a given vector field and the boundary integral of the inner product of said field with the geometric measure theoretic outward unit normal to the underlying domain. In order to state it, a number of definitions and preliminary results are discussed.

Given an open set $\Omega \subseteq \mathbb{R}^{n}$ and an aperture parameter $\kappa \in(0, \infty)$, define the nontangential approach regions

$$
\Gamma_{\mathcal{K}}(x):=\{y \in \Omega:|y-x|<(1+\kappa) \operatorname{dist}(y, \partial \Omega)\}
$$

for each $x \in \partial \Omega$. In turn, these regions are used to define the nontangential maximal operator $\mathcal{N}_{\kappa}$, acting on each $\mathcal{L}^{n}$-measurable function $u$ defined in $\Omega$ according to ${ }^{2}$

$$
\left(\mathcal{N}_{\mathcal{K}} u\right)(x):=\|u\|_{L^{\infty}\left(\Gamma_{\mathcal{K}}(x), \mathcal{L}^{n}\right)} \text { for each } x \in \partial \Omega .
$$

Note that if we work (as one usually does) with equivalence classes, obtained by identifying functions which coincide $\mathcal{L}^{n}$-a.e., the nontangential maximal operator is independent of the specific choice of a representative in a given equivalence class. More generally, if $u: \Omega \rightarrow \mathbb{R}$ is a Lebesgue measurable function and $E \subseteq \Omega$ is an arbitrary $\mathcal{L}^{n}$-measurable set, we denote by $\mathcal{N}_{\kappa}^{E} u$ the nontangential maximal function of $u$ restricted to $E$, that is, $\mathcal{N}_{\mathcal{K}}^{E} u: \partial \Omega \rightarrow[0,+\infty]$ defined as

$$
\left(\mathcal{N}_{\mathcal{K}}^{E} u\right)(x):=\|u\|_{L^{\infty}\left(\Gamma_{\mathcal{K}}(x) \cap E, \mathcal{L}^{n}\right)} \text { for each } x \in \partial \Omega .
$$

Definition 2.1. Fix a background parameter $\kappa>0$ and let $u$ be a complex-valued Lebesgue measurable function defined $\mathcal{L}^{n}$-a.e. in an open set $\Omega \subset \mathbb{R}^{n}$. Consider a point $x \in \partial \Omega$ such that $x \in \overline{\Gamma_{\mathcal{K}}(x)}$. The $x$-nontangential limit of $u$ at $x$ from within $\Gamma_{k}(x)$ is said to exist, and its value is the number $a \in \mathbb{C}$, provided

for every $\varepsilon>0$ there exists $r>0$ so that $|u(y)-a|<\varepsilon$ for $\mathcal{L}^{n}$-a.e. $y \in \Gamma_{\mathcal{k}}(x) \cap B(x, r)$.

Whenever the $x$-nontangential limit of $u$ at $x$ from within $\Gamma_{\mathcal{K}}(x)$ exists, its value is denoted by $\left(\left.u\right|_{\partial \Omega} ^{k-\text { n.t. }}\right)(x)$.

Definition 2.2. Let $\Sigma \subseteq \mathbb{R}^{n}$ be a closed set.

(i) $\Sigma$ is said to be lower Ahlfors regular provided there exists a constant $c \in(0, \infty)$ such that

$$
c r^{n-1} \leq \mathcal{H}^{n-1}(B(x, r) \cap \Sigma)
$$

for each $x \in \Sigma$ and $r \in(0,2 \operatorname{diam}(\Sigma))$.

\footnotetext{
${ }^{2}$ We make the convention that $\left(\mathcal{N}_{\mathcal{K}} u\right)(x):=0$ whenever $x \in \partial \Omega$ is such that $\Gamma_{\mathcal{K}}(x)=\emptyset$.
}

(ii) $\Sigma$ is said to be upper Ahlfors regular if there exists a constant $C \in(0, \infty)$ with the property that

$$
\begin{gathered}
\mathcal{H}^{n-1}(B(x, r) \cap \Sigma) \leq C r^{n-1} \\
\text { for each } x \in \Sigma \text { and } r>0 .
\end{gathered}
$$

(iii) $\Sigma$ is said to be Ahlfors regular ${ }^{3}$ if it is both lower and upper Ahlfors regular.

Definition 2.3. Given an arbitrary nonempty open proper subset $\Omega$ of $\mathbb{R}^{n}$, define its nontangentially accessible boundary as

$$
\partial_{\mathrm{nta}} \Omega:=\left\{x \in \partial \Omega: x \in \overline{\Gamma_{\mathcal{K}}(x)} \text { for each } \kappa>0\right\} .
$$

A basic result proved in [17] states that if $\Omega \subseteq \mathbb{R}^{n}$ is an open set with a lower Ahlfors regular boundary and such that the measure $\sigma:=\mathcal{H}^{n-1}\left\lfloor\partial \Omega\right.$ is doubling, ${ }^{4}$ then $\sigma\left(\partial_{*} \Omega \backslash \partial_{\text {nta }} \Omega\right)=0$; that is, the set $\partial_{\text {nta }} \Omega$ covers $\partial_{*} \Omega$ up to a $\sigma$-nullset.

Hypothesis 2.4. Fix $n \in \mathbb{N}$ and let $\Omega$ be a nonempty, proper, open subset of $\mathbb{R}^{n}$, with a lower Ahlfors regular boundary such that $\sigma:=\mathcal{H}^{n-1}[\partial \Omega$ is a doubling measure. Throughout, the symbol $\nu$ is reserved for the geometric measure theoretic outward unit normal to $\Omega$.

In the context of Hypothesis $2.4, \Omega$ is a set of locally finite perimeter, so $\nu$ is defined $\sigma$-a.e. on $\partial_{*} \Omega$. Also, if $\partial \Omega$ is actually Ahlfors regular, then automatically $\sigma$ is doubling. Here is the actual statement of the theorem alluded to earlier:

Theorem 2.5. Assume Hypothesis 2.4. Fix $\kappa \in(0, \infty)$ and assume that $\vec{F}=\left(F_{1}, \ldots, F_{n}\right): \Omega \rightarrow \mathbb{C}^{n}$ is a vector field with Lebesgue measurable components, satisfying

$$
\begin{gathered}
\left.\vec{F}\right|_{\partial \Omega} ^{k-n . t .} \text { exists }\left(\text { in } \mathbb{C}^{n}\right) \text { at } \sigma \text {-a.e. point on } \partial_{\mathrm{nta}} \Omega, \\
\mathcal{N}_{\mathcal{K}} \vec{F} \text { belongs to the space } L^{1}(\partial \Omega, \sigma) \text {, and } \\
\operatorname{div} \vec{F}:=\partial_{1} F_{1}+\cdots+\partial_{n} F_{n} \in L^{1}\left(\Omega, \mathcal{L}^{n}\right),
\end{gathered}
$$

where all partial derivatives are considered in the sense of distributions in $\Omega$.

Then for any $\kappa^{\prime}>0$ the nontangential trace $\left.\vec{F}\right|_{\partial \Omega} ^{\kappa^{\prime}-\text { n.t. }}$ exists $\sigma$-a.e. on $\partial_{\mathrm{nta}} \Omega$ and is actually independent of $\mathcal{K}^{\prime}$. When regarding it as a function defined $\sigma$-a.e. on $\partial_{*} \Omega$ (which, up to a $\sigma$-nullset, is contained in $\partial_{n t a} \Omega$ ), this nontangential trace belongs to $\left[L^{1}\left(\partial_{*} \Omega, \sigma\right)\right]^{n}$. Also, with the dependence on the parameter $\kappa^{\prime}$ dropped, one has

$$
\int_{\Omega} \operatorname{div} \vec{F} d \mathcal{L}^{n}=\int_{\partial_{*} \Omega} \nu \cdot\left(\left.\vec{F}\right|_{\partial \Omega} ^{\text {n.t. }}\right) d \sigma
$$

when either $\Omega$ is bounded, or $\partial \Omega$ is unbounded and $n \geq 2$. In the remaining cases, i.e., when $\Omega$ is unbounded, and either $\partial \Omega$

\footnotetext{
${ }^{3}$ Often, this is referred to as Ahlfors-David regular, or ADR for short.

${ }^{4}$ That is, there exists some $C \in[1, \infty)$ such that $0<\sigma(B(x, 2 r) \cap \partial \Omega) \leq$ $C \sigma(B(x, r) \cap \partial \Omega)<+\infty$ for all $x \in \partial \Omega$ and $r \in(0, \infty)$.
} 
is bounded or $n=1$, formula (2.6) continues to hold under the additional assumption that there exists $\lambda \in(1, \infty)$ such that

$$
\begin{gathered}
\int_{A_{\lambda, R} \cap \Omega}|x \cdot \vec{F}(x)| d \mathcal{L}^{n}(x)=o\left(R^{2}\right) \text { as } R \rightarrow \infty, \\
\text { where } A_{\lambda, R}:=B(0, \lambda R) \backslash B(0, R) .
\end{gathered}
$$

This result refines work in [11], [15], [18], [19], where earlier versions, interesting in their own right, along with proofs and a wealth of applications may be found. The current, sharp version, appears in [17].

As expected, Theorem 2.5 contains (1.2) as a special case. More generally, the scenario in which $\Omega$ is a bounded Lipschitz domain in $\mathbb{R}^{n}$ and $\vec{F} \in\left[\mathcal{C}^{0}(\bar{\Omega})\right]^{n}$ is a vector field which is differentiable at every point in $\Omega$ and $\sum_{j=1}^{n} \partial_{j} F_{j}$ (where the partial derivatives are considered in a pointwise, classical sense) is continuous and absolutely integrable on $\Omega$ is also covered by Theorem 2.5. Significantly, Theorem 2.5 contains (when $n=1$ ) the sharp form of the Fundamental Theorem of Calculus recalled in (1.1).

Going further, observe that absolutely integrable functions in an open subset $\Omega$ of $\mathbb{R}^{n}$ may be identified with complex Borel measures in $\Omega$ (the collection of which is henceforth denoted by $\operatorname{CBM}(\Omega)$ ) via

$$
L^{1}\left(\Omega, \mathcal{L}^{n}\right) \ni f \longmapsto f \mathcal{L}^{n} \in \operatorname{CBM}(\Omega) .
$$

A useful generalization of Theorem 2.5 is obtained by replacing the last condition in (2.5) with the requirement that

the distribution $\operatorname{div} \vec{F}:=\sum_{j=1}^{n} \partial_{j} F_{j} \in \mathcal{D}^{\prime}(\Omega)$

extends to a complex Borel measure in $\Omega$.

Retaining the symbol $\operatorname{div} \vec{F}$ for the said extension, formula (2.12) takes the form

$$
(\operatorname{div} \vec{F})(\Omega)=\int_{\partial_{*} \Omega} \nu \cdot\left(\left.\vec{F}\right|_{\partial \Omega} ^{\text {n.t. }}\right) d \sigma .
$$

We can also dispense with the decay condition (2.7) at the expense of an additional term, encoding information about the behavior of $\vec{F}$ at infinity. Specifically, if one fixes some $\phi \in \mathcal{C}_{c}^{\infty}\left(\mathbb{R}^{n}\right)$ satisfying $\phi \equiv 1$ near the origin in $\mathbb{R}^{n}$, it follows that the limit

$$
[\vec{F}]_{\infty}:=\lim _{R \rightarrow \infty}\left\{\frac{1}{R} \int_{\Omega}(\nabla \phi)(x / R) \cdot \vec{F}(x) d \mathcal{L}^{n}(x)\right\}
$$

(henceforth termed the contribution of $\vec{F}$ at infinity) exists and

$$
(\operatorname{div} \vec{F})(\Omega)=\int_{\partial_{*} \Omega} \nu \cdot\left(\left.\vec{F}\right|_{\partial \Omega} ^{\text {n.t. }}\right) d \sigma-[\vec{F}]_{\infty} .
$$

Once the possibility of allowing the divergence of the vector field to belong to a class larger than the collection of integrable functions is considered, other natural options present themselves. To discuss an important case of this flavor, we start by observing that, given a nonempty open set $\Omega \subseteq \mathbb{R}^{n}$, one has the injective embeddings ${ }^{5}$

$$
\begin{gathered}
\mathcal{E}^{\prime}(\Omega) \hookrightarrow \mathcal{D}^{\prime}(\Omega) \text { and } \\
L^{1}\left(\Omega, \mathcal{L}^{n}\right) \hookrightarrow \operatorname{CBM}(\Omega) \hookrightarrow \mathcal{D}^{\prime}(\Omega) .
\end{gathered}
$$

In view of these embeddings, it makes sense to consider the subspace $\mathcal{E}^{\prime}(\Omega)+\operatorname{CBM}(\Omega)$ of $\mathcal{D}^{\prime}(\Omega)$ defined as the collection of those $u \in \mathcal{D}^{\prime}(\Omega)$ for which there exist some $w \in \mathcal{E}^{\prime}(\Omega)$ and $\mu \in \operatorname{CBM}(\Omega)$ such that $u=w+\mu$ in $\mathcal{D}^{\prime}(\Omega)$. We also introduce the space of smooth, bounded, complex-valued functions in $\Omega$, i.e.,

$$
\mathcal{C}_{b}^{\infty}(\Omega):=\left\{f \in \mathcal{C}^{\infty}(\Omega): f \text { bounded in } \Omega\right\},
$$

and denote by $\left(\mathcal{C}_{b}^{\infty}(\Omega)\right)^{*}$ the algebraic dual of this linear space. Throughout, we shall use $X^{*}(\cdot, \cdot)_{X}$ to denote the duality pairing between a linear space $X$ and its algebraic dual $X^{*}$.

Theorem 2.6. Assume Hypothesis 2.4. Fix $\kappa \in(0, \infty)$ and assume that the vector field

$$
\vec{F}=\left(F_{1}, \ldots, F_{n}\right) \in\left[\mathcal{D}^{\prime}(\Omega)\right]^{n}
$$

is such that there exists a compact set $K$ contained in $\Omega$ satisfying

$$
\begin{gathered}
\left.\vec{F}\right|_{\Omega \backslash K} \in\left[L_{\text {loc }}^{1}\left(\Omega \backslash K, \mathcal{L}^{n}\right)\right]^{n} \text { and } \\
\mathcal{N}_{\mathcal{K}}^{\Omega \backslash K} \vec{F} \in L_{\text {loc }}^{1}(\partial \Omega, \sigma) .
\end{gathered}
$$

In addition, assume that the pointwise nontangential boundary trace

$$
\left.\vec{F}\right|_{\partial \Omega} ^{\kappa-\text { n.t. }}=\left(\left.F_{1}\right|_{\partial \Omega} ^{\kappa-\text { n.t. }}, \ldots,\left.F_{n}\right|_{\partial \Omega} ^{\kappa-\text { n.t. }}\right)
$$

exists $\left(\right.$ in $\left.\mathbb{C}^{n}\right)$ at $\sigma$-a.e. point on $\partial_{\mathrm{nta}} \Omega$, has the property that

$$
\nu \cdot\left(\left.\vec{F}\right|_{\partial \Omega} ^{k-\text { n.t. }}\right) \in L^{1}\left(\partial_{*} \Omega, \sigma\right)
$$

and the divergence of $\vec{F}$, taken in the sense of distributions in $\Omega$, is the sum (in $\mathcal{D}^{\prime}(\Omega)$ ) of a compactly supported distribution in $\Omega$ and a complex Borel measure in $\Omega$, i.e.,

$$
\operatorname{div} \vec{F} \in \mathcal{E}^{\prime}(\Omega)+\operatorname{CBM}(\Omega) .
$$

Then for any $\kappa^{\prime}>0$ the nontangential trace $\left.\vec{F}\right|_{\partial \Omega} ^{\kappa^{\prime}-\text { n.t. }}$ exists $\sigma$-a.e. on $\partial_{\text {nta }} \Omega$ and is actually independent of $\kappa^{\prime}$. In addition, with the dependence on the parameter $\mathcal{K}^{\prime}$ dropped, one has

$$
\left(\mathcal{C}_{b}^{\infty}(\Omega)\right)^{*}(\operatorname{div} \vec{F}, 1)_{\mathcal{C}_{b}^{\infty}(\Omega)}=\int_{\partial_{*} \Omega} v \cdot\left(\left.\vec{F}\right|_{\partial \Omega} ^{\text {n.t. }}\right) d \sigma-[\vec{F}]_{\infty} .
$$

Also, the contribution at infinity vanishes, i.e., $[\vec{F}]_{\infty}=0$, whenever there exists $\lambda \in(1, \infty)$ such that

$$
\begin{gathered}
\int_{A_{\lambda, R} \cap \Omega}|x \cdot \vec{F}(x)| d \mathcal{L}^{n}(x)=o\left(R^{2}\right) \text { as } R \rightarrow \infty, \\
\text { where } A_{\lambda, R}:=B(0, \lambda R) \backslash B(0, R) .
\end{gathered}
$$

${ }^{5}$ Recall that $\mathcal{E}^{\prime}(\Omega)$ is the subspace of $\mathcal{D}^{\prime}(\Omega)$ consisting of all compactly supported distributions in $\Omega$. 
In such a scenario, the above Divergence Formula reduces to

$$
\left(\mathcal{C}_{b}^{\infty}(\Omega)\right)^{*}(\operatorname{div} \vec{F}, 1)_{\mathcal{C}_{b}^{\infty}(\Omega)}=\int_{\partial_{*} \Omega} v \cdot\left(\left.\vec{F}\right|_{\partial \Omega} ^{\text {n.t. }}\right) d \sigma .
$$

Finally, condition (2.19) is automatically satisfied when either $\Omega$ is bounded, or

$$
\begin{aligned}
& \partial \Omega \text { is unbounded, } n \geq 2, \\
& \text { and } \mathcal{N}_{\mathcal{K}}^{\Omega \backslash K} \vec{F} \in L^{1}(\partial \Omega, \sigma) .
\end{aligned}
$$

Hence, (2.20) holds in either of these cases.

Theorem 2.6 is proved employing a localization argument aimed at decomposing the given vector field into a regular part, to which Theorem 2.5 applies, plus a singular part which has compact support and, as such, can be handled directly (using distribution theory; cf. [16]).

There is also a version of Theorem 2.6 formulated in an open set $\Omega \subseteq \mathbb{R}^{n}$ without imposing the condition that the "surface measure" $\sigma:=\mathcal{H}^{n-1}[\partial \Omega$ is doubling. Remarkably, there is only a relatively small price to pay in this scenario, namely, the loss of flexibility in the choice of the aperture parameter $\kappa \in(0, \infty)$ used to define the nontangential approach regions entering the definition of the nontangential boundary trace $\left.\vec{F}\right|_{\partial \Omega} ^{\kappa \text {-n.t. }}$ and the nontangential maximal function $\mathcal{N}_{\mathcal{K}} \vec{F}$ for the given vector field $\vec{F}$. At the same time, we may further relax the demand made in (2.18) on the nature of the distribution $\operatorname{div} \vec{F}$ by now merely asking that this be extended to a functional in the algebraic dual $\left(\mathcal{C}_{b}^{\infty}(\Omega)\right)^{*}$ exhibiting a mild, natural, continuity property (that is automatically satisfied when (2.18) holds).

We also wish to note that the results presented here are robust, as they continue to work in other settings of interest. For example, we have a natural version of the classical Stokes theorem for differential forms on manifolds in which the pullback of the given form to the boundary is now interpreted as a suitable Radon measure.

\section{Some Applications}

The Divergence Theorems presented earlier share a number of common features. For example, all vector fields involved may lack any type of continuity, and their boundary traces are taken in a nontangential pointwise fashion. The rationale for insisting on such characteristics may be traced back to classical results in harmonic analysis, complex analysis, partial differential equations, and potential theory (specifically, the theory of Hardy spaces, Fatou-type theorems, elliptic boundary value problems, and Calderón-Zygmund theory for singular integral operators, among others). In turn, this innate affinity with the very nature of this body of mathematics makes our brand of Divergence Theorem an effective tool in dealing with problems in these areas. A vast number of applications are discussed at length in the monograph [17]. Here we limit ourselves to presenting a couple of applications of general appeal.

First, we consider a versatile version of the integration by parts formula for first-order operators. To facilitate its statement, given any constant coefficient first-order system $D=\sum_{j} A_{j} \partial_{j}+B$ we agree to define its (real) transpose as the operator $D^{\top}:=-\sum_{j} A_{j}^{\top} \partial_{j}+B^{\top}$, and its principal symbol as the matrix-valued function associating to each $\xi=\left(\xi_{j}\right)_{j} \in \mathbb{R}^{n}$ the matrix $\operatorname{Sym}(D ; \xi)=\sum_{j} \xi_{j} A_{j}$.

Theorem 3.1. Assume Hypothesis 2.4. Let $D$ be an $N \times M$ first-order system with constant complex coefficients in $\mathbb{R}^{n}$, and let $u: \Omega \rightarrow \mathbb{C}^{M}, w: \Omega \rightarrow \mathbb{C}^{N}$ be Lebesgue measurable functions satisfying, for some $\kappa, \kappa^{\prime}>0$,

$\mathcal{N}_{\mathcal{K}} u<\infty$ and $\mathcal{N}_{\mathcal{K}^{\prime}}, w<\infty$ at $\sigma$-a.e. point on $\partial \Omega$,

$\mathcal{N}_{\mathcal{K}} u \cdot \mathcal{N}_{\mathcal{K}^{\prime}} w$ belongs to the space $L^{1}(\partial \Omega, \sigma)$,

$\left.u\right|_{\partial \Omega} ^{\kappa-\text { n.t. }},\left.w\right|_{\partial \Omega} ^{\kappa^{\prime}-\text {-n.t. }}$ exist at $\sigma$-a.e. point on $\partial_{\mathrm{nta}} \Omega$,

$D u \in\left[L_{\text {loc }}^{1}\left(\Omega, \mathcal{L}^{n}\right)\right]^{N}, \quad D^{\top} w \in\left[L_{\text {loc }}^{1}\left(\Omega, \mathcal{L}^{n}\right)\right]^{M}$,

$\langle D u, w\rangle \in L^{1}\left(\Omega, \mathcal{L}^{n}\right)$ and $\left\langle u, D^{\top} w\right\rangle \in L^{1}\left(\Omega, \mathcal{L}^{n}\right)$.

Then for any $\kappa^{\prime \prime}>0$ the nontangential traces $\left.u\right|_{\partial \Omega} ^{\kappa^{\prime \prime}-\text { n.t. }}$, $\left.w\right|_{\partial \Omega} ^{x^{\prime \prime}-\text { n.t. }}$ exist $\sigma$-a.e. on $\partial_{\mathrm{nta}} \Omega$ and are actually independent of $\kappa^{\prime \prime}$. When regarding them as functions defined $\sigma$-a.e. on $\partial_{*} \Omega$ and dropping the dependence on the parameter $\kappa^{\prime \prime}$, the following integration by parts formula, involving absolutely convergent integrals, holds:

$$
\begin{aligned}
\int_{\Omega}\langle D u, w\rangle d \mathcal{L}^{n}= & \int_{\Omega}\left\langle u, D^{\top} w\right\rangle d \mathcal{L}^{n} \\
& +\int_{\partial_{*} \Omega}\left\langle\operatorname{Sym}(D ; \nu)\left(\left.u\right|_{\partial \Omega} ^{\text {n.t. }}\right),\left.w\right|_{\partial \Omega} ^{\text {n.t. }}\right\rangle d \sigma
\end{aligned}
$$

when either $\Omega$ is bounded, or $\partial \Omega$ is unbounded and $n \geq 2$. Furthermore, formula (3.1) also holds if $\Omega$ is unbounded, and either $\partial \Omega$ is bounded or $n=1$, provided there exists $\lambda \in(1, \infty)$ such that

$$
\begin{gathered}
\int_{A_{\lambda, R} \cap \Omega}|u \| w| d \mathcal{L}^{n}=o(R) \text { as } R \rightarrow \infty, \\
\text { where } A_{\lambda, R}:=B(0, \lambda R) \backslash B(0, R) .
\end{gathered}
$$

Of course, some of the most familiar choices for $D$ are the gradient, curl, and divergence operators. Also, a closely related version of this theorem holds on Riemannian manifolds, where natural choices for $D$ include the exterior derivative operator, the deformation tensor, the Levi-Civita connection, etc. (see the discussion in [18] on this topic). Regarding the optimality of Theorem 3.1, a glimpse is offered by considering the very special case when $n=1, \Omega=(a, b) \subset \mathbb{R}$, and $D=d / d x$. Then Theorem 3.1 asserts that for any functions $u, w \in L^{\infty}(a, b)$ with 
$u^{\prime}, w^{\prime} \in L_{\mathrm{loc}}^{1}(a, b)$, such that $u^{\prime} w$ and $u w^{\prime}$ are in $L^{1}(a, b)$, and the limits $u\left(a^{+}\right):=\lim _{x \rightarrow a^{+}} u(x), w\left(a^{+}\right):=\lim _{x \rightarrow a^{+}} w(x)$, $u\left(b^{-}\right):=\lim _{x \rightarrow b^{-}} u(x), w\left(b^{-}\right):=\lim _{x \rightarrow b^{-}} w(x)$ exist, we have

$$
\int_{a}^{b} u^{\prime} w d x=u\left(b^{-}\right) w\left(b^{-}\right)-u\left(a^{+}\right) w\left(a^{+}\right)-\int_{a}^{b} u w^{\prime} d x .
$$

The special case when $u \in \mathrm{AC}([a, b])$ and $w=1$ yields the sharp Fundamental Theorem of Calculus stated in (1.1).

Sketch of proof of Theorem 3.1. If $D=\sum_{j=1}^{n} A_{j} \partial_{j}+B$, then the idea is to apply Theorem 2.5 to the vector field $\vec{F}=\left(F_{j}\right)_{1 \leq j \leq n}$, whose $j$ th component is $F_{j}:=\left\langle A_{j} u, w\right\rangle$ for each $j \in\{1, \ldots, n\}$. Upon checking that $\operatorname{div} \vec{F}=$ $\langle D u, w\rangle-\left\langle u, D^{\top} w\right\rangle$ in $\mathcal{D}^{\prime}(\Omega)$ and that $\left.\vec{F}\right|_{\partial \Omega} ^{k-\text { n.t. }}$ is given by $\left\langle\operatorname{Sym}(D ; v)\left(\left.u\right|_{\partial \Omega} ^{k-\text { n.t. }}\right),\left.w\right|_{\partial \Omega} ^{k \text {-n.t. }}\right\rangle$ at $\sigma$-a.e. point on $\partial_{\text {nta }} \Omega$, the formula claimed in (3.1) is implied by (2.6).

Our second application is a very general version of the Cauchy-Pompeiu representation formula. To state it, we first recall that the Cauchy-Riemann operator $\bar{\partial}$ in the plane and its conjugate are, respectively, defined as

$$
\bar{\partial}:=\frac{1}{2}\left(\partial_{x}+i \partial_{y}\right) \text { and } \partial:=\frac{1}{2}\left(\partial_{x}-i \partial_{y}\right)
$$

where $i:=\sqrt{-1} \in \mathbb{C}$. Given any Lebesgue measurable set $\Omega \subseteq \mathbb{R}^{2} \equiv \mathbb{C}$ of locally finite perimeter, we agree to define the complex arc-length measure on $\partial \Omega$ by

$$
d \zeta:=-2 i \bar{\partial} \mathbf{1}_{\Omega},
$$

where the derivatives are taken in the sense of distributions in $\mathbb{R}^{2}$. Hence, if $\sigma:=\mathcal{H}^{1}[\partial \Omega$ and $\nu$ denotes the geometric measure theoretic outward unit normal to $\Omega$ (canonically identified with a complex-valued measure), it follows that the complex arc-length measure on $\partial \Omega$ is supported on $\partial_{*} \Omega$ and satisfies

$$
d \zeta=i \nu(\zeta) d \sigma(\zeta) \text { on } \partial_{*} \Omega .
$$

Theorem 3.2. Let $\Omega \subseteq \mathbb{R}^{2} \equiv \mathbb{C}$ be an open set with a lower Ahlfors regular boundary, such that $\sigma:=\mathcal{H}^{1}[\partial \Omega$ is a doubling measure on $\partial \Omega$. In this context, suppose $u: \Omega \rightarrow \mathbb{C}$ is an $\mathcal{L}^{2}$-measurable complex-valued function which, for some $\kappa>0$, satisfies

$$
\begin{gathered}
\int_{\partial \Omega} \frac{\left(\mathcal{N}_{\mathcal{K}} u\right)(\zeta)}{1+|\zeta|} d \sigma(\zeta)<+\infty, \\
\text { and }\left.u\right|_{\partial \Omega} \text {-n.t. } \\
\text { exists } \sigma \text {-a.e. on } \partial_{\mathrm{nta}} \Omega \text {. }
\end{gathered}
$$

In addition, with the Cauchy-Riemann operator $\bar{\partial}$ taken in the sense of distributions in $\Omega$, assume that

$$
\bar{\partial} u \in L^{1}\left(\Omega, \frac{d \mathcal{L}^{2}(\zeta)}{1+|\zeta|}\right)
$$

Then for any $\kappa^{\prime}>0$ the nontangential trace $\left.u\right|_{\partial \Omega} ^{\kappa^{\prime} \text {-n.t. }}$ exists at $\sigma$-a.e. point on $\partial_{n t a} \Omega$ and is actually independent of $\kappa^{\prime}$. Moreover, with the dependence on the parameter $\kappa^{\prime}$ dropped, for $\mathcal{L}^{2}$-a.e. point $z \in \Omega$ one has (with absolutely convergent integrals)

$$
\begin{aligned}
u(z)=\frac{1}{2 \pi i} \int_{\partial_{*} \Omega} \frac{\left(\left.u\right|_{\partial \Omega} ^{\text {n.t. }}\right)(\zeta)}{\zeta-z} d \zeta \\
\\
\quad-\frac{1}{\pi} \int_{\Omega} \frac{(\bar{\partial} u)(\zeta)}{\zeta-z} d \mathcal{L}^{2}(\zeta),
\end{aligned}
$$

provided $\Omega$ is bounded, or $\partial \Omega$ is unbounded. In the remaining case, i.e., when $\Omega$ is unbounded and $\partial \Omega$ is bounded (that is, when $\Omega$ is an exterior domain), formula (3.8) holds under the additional assumption that there exists $\lambda \in(1, \infty)$ such that

$$
\begin{aligned}
& f_{A_{\lambda, R}}|u| d \mathcal{L}^{2}=o(1) \text { as } R \rightarrow \infty, \\
& \text { where } A_{\lambda, R}:=B(0, \lambda R) \backslash B(0, R) .
\end{aligned}
$$

As a corollary, if $\bar{\partial} u=0$ in $\Omega$, then one has the Cauchy integral representation formula

$$
u(z)=\frac{1}{2 \pi i} \int_{\partial_{*} \Omega} \frac{\left(\left.u\right|_{\partial \Omega} ^{\text {n.t. }}\right)(\zeta)}{\zeta-z} d \zeta, \quad \forall z \in \Omega,
$$

provided $\Omega$ is bounded, or $\partial \Omega$ is unbounded. In addition, when $\Omega$ is unbounded and $\partial \Omega$ is bounded, formula (3.10) continues to be valid provided (3.9) is also assumed.

Proof. That for any $\kappa^{\prime}>0$ the nontangential trace $\left.u\right|_{\partial \Omega} ^{\kappa^{\prime}-\text { n.t. }}$ exists $\sigma$-a.e. on $\partial_{\text {nta }} \Omega$ and is independent of $\kappa^{\prime}$ is proved in [17]. We make two observations. One is that the first property in (3.6) entails

$$
u \in L_{\mathrm{loc}}^{\infty}\left(\Omega, \mathcal{L}^{2}\right) .
$$

The other one is that (3.7) implies that for $\mathcal{L}^{2}$-a.e. $z \in \mathbb{C}$ we have

$$
\int_{\Omega}\left|\frac{(\bar{\partial} u)(\zeta)}{\zeta-z}\right| d \mathcal{L}^{2}(\zeta)<+\infty .
$$

Indeed, since elementary considerations show that there exists a constant $C \in(0, \infty)$ such that for each $R \in(0, \infty)$ we have

$$
\int_{B(0, R)} \frac{d \mathcal{L}^{2}(z)}{|z-\zeta|} \leq C \cdot \frac{R^{2}}{R+|\zeta|} \text { for all } \zeta \in \mathbb{C}
$$

for each $R \in(0, \infty)$ one can use the Fubini-Tonelli 
Theorem to write

$$
\begin{aligned}
\int_{B(0, R)} & \left(\int_{\Omega} \frac{|(\bar{\partial} u)(\zeta)|}{|\zeta-z|} d \mathcal{L}^{2}(\zeta)\right) d \mathcal{L}^{2}(z) \\
& =\int_{\Omega}|(\bar{\partial} u)(\zeta)|\left(\int_{B(0, R)} \frac{d \mathcal{L}^{2}(z)}{|\zeta-z|}\right) d \mathcal{L}^{2}(\zeta) \\
& \leq C_{R} \int_{\Omega} \frac{|(\bar{\partial} u)(\zeta)|}{1+|\zeta|} d \mathcal{L}^{2}(\zeta)<+\infty
\end{aligned}
$$

for some constant $C_{R} \in(0, \infty)$. In turn, (3.14) implies that (3.13) holds for $\mathcal{L}^{2}$-a.e. point $z \in B(0, R)$, and the desired conclusion follows on account of the arbitrariness of $R$.

Next, fix a Lebesgue point $z \in \Omega$ of $u$ with the property that (3.12) holds, and define the vector field

$$
\vec{F}_{z}(\zeta):=\left(\frac{u(\zeta)}{\zeta-z}, i \frac{u(\zeta)}{\zeta-z}\right) \text { for } \mathcal{L}^{2} \text {-a.e. } \zeta \in \Omega \text {. }
$$

Then from (3.15) and (3.11) one concludes that $\vec{F}_{z}$ belongs to $\left[L_{\text {loc }}^{1}\left(\Omega, \mathcal{L}^{2}\right)\right]^{2}$. Also, with $\delta_{z}$ denoting the Dirac distribution with mass at $z$, we have

$$
\begin{aligned}
\operatorname{div} \vec{F}_{z}(\zeta) & =2 \bar{\partial}_{\zeta}\left[\frac{u(\zeta)}{\zeta-z}\right] \\
& =2 \pi u(z) \delta_{z}+2 \frac{(\bar{\partial} u)(\zeta)}{\zeta-z} \text { in } \mathcal{D}^{\prime}(\Omega),
\end{aligned}
$$

where the last equality is a slight extension of [16, Exercise 7.47, p. 292]. In particular, (3.16) and (3.12) imply that

$$
\operatorname{div} \vec{F}_{z} \text { belongs to } \mathcal{E}^{\prime}(\Omega)+L^{1}\left(\Omega, \mathcal{L}^{2}\right) .
$$

If we now consider $K:=\overline{B(z \text {, dist }(z, \partial \Omega) / 2)}$, then $K$ is a compact set contained in $\Omega$ and (3.15) allows us to estimate

$$
\left(\mathcal{N}_{\mathcal{K}}^{\Omega \backslash K} \vec{F}_{z}\right)(\zeta) \leq C_{z} \frac{\left(\mathcal{N}_{\mathcal{K}} u\right)(\zeta)}{|\zeta-z|} \text { for all } \zeta \in \partial \Omega
$$

In turn, from (3.18) and the first condition in (3.6) we conclude that $\mathcal{N}_{\mathcal{K}}^{\Omega \backslash K} \vec{F}_{z} \in L^{1}(\partial \Omega, \sigma)$. Moreover, (3.15) and the assumptions on $u$ imply that $\left.\vec{F}_{z}\right|_{\partial \Omega} ^{\kappa-\text { n.t. }}$ exists $\sigma$-a.e. in $\partial_{\text {nta }} \Omega$ and, in fact, that

$$
\begin{gathered}
\left(\left.\vec{F}_{z}\right|_{\partial \Omega} ^{\kappa-\text { n.t. }}\right)(\zeta)=\left(\frac{\left(\left.u\right|_{\partial \Omega} ^{\kappa-\text { n.t. }}\right)(\zeta)}{\zeta-z}, i \frac{\left(\left.u\right|_{\partial \Omega} ^{\kappa-\text { n.t. }}\right)(\zeta)}{\zeta-z}\right) \\
\text { for } \sigma \text {-a.e. } \zeta \in \partial_{\text {nta }} \Omega .
\end{gathered}
$$

Finally, observe that in the case when $\Omega$ is an exterior domain, condition (3.9) implies that

$$
\int_{A_{\lambda, R}}\left|\vec{F}_{z}\right| d \mathcal{L}^{2}=o(R) \text { as } R \rightarrow \infty .
$$

In summary, we have proved that $\vec{F}_{z}$ satisfies all hypotheses in Theorem 2.6 whenever $z \in \Omega$ is a Lebesgue point of $u$ with the property that (3.12) holds. In combination with (3.16) and (3.19) (and also assuming (3.9) in the case when $\Omega$ is an exterior domain), for each such point $z$ this permits us to write

$$
\begin{aligned}
2 \pi & u(z)+2 \int_{\Omega} \frac{(\bar{\partial} u)(\zeta)}{\zeta-z} d \mathcal{L}^{2}(\zeta) \\
& =\left(\mathcal{e}_{b}^{\infty}(\Omega)\right)^{*}\left(\operatorname{div} \vec{F}_{z}, 1\right)_{\mathcal{C}_{b}^{\infty}(\Omega)}=\int_{\partial_{*} \Omega}\left\langle\nu,\left.\vec{F}_{z}\right|_{\partial \Omega} ^{\text {n.t. }}\right\rangle d \sigma \\
& =\int_{\partial_{*} \Omega}\left\langle\nu(\zeta),\left(\frac{\left(\left.u\right|_{\partial \Omega} ^{\text {n.t. }}\right)(\zeta)}{\zeta-z}, i \frac{\left(\left.u\right|_{\partial \Omega} ^{\text {n.t. }}\right)(\zeta)}{\zeta-z}\right)\right\rangle d \sigma(\zeta) \\
& =\int_{\partial_{*} \Omega} \frac{\left(\left.u\right|_{\partial \Omega} ^{\text {n.t. }}\right)(\zeta)}{\zeta-z} \nu(\zeta) d \sigma(\zeta) \\
= & \frac{1}{i} \int_{\partial_{*} \Omega} \frac{\left(\left.u\right|_{\partial \Omega} ^{\text {n.t. }}\right)(\zeta)}{\zeta-z} d \zeta
\end{aligned}
$$

where the last equality uses (3.5). From this (3.8) follows at each Lebesgue point $z \in \Omega$ of $u$ such that (3.12) holds, hence at $\mathcal{L}^{2}$-a.e. point $z \in \Omega$.

It turns out that the doubling assumption on the measure $\sigma:=\mathcal{H}^{1}\lfloor\partial \Omega$ may be relaxed to simply asking that $\sigma$ be a locally finite measure on $\partial \Omega$. The price to pay is having to demand that the aperture parameter $\kappa$ be sufficiently large (depending on $\Omega$ ) to begin with, and we may lose the flexibility of changing it when considering nontangential boundary traces. Modulo these nuances, the format of the main result (i.e., the integral representation formula (3.8)) remains the same.

This being said, the lower Ahlfors regularity condition for $\partial \Omega$ may not be simply dropped. To see this, consider the open subset $\Omega:=B(0,1) \backslash\{0\}$ of $\mathbb{C}$ and the function $u: \Omega \rightarrow \mathbb{C}$ defined as $u(\zeta):=\frac{1}{\zeta}$ for each $\zeta \in \Omega$. It is clear that $\sigma:=\mathcal{H}^{1}\lfloor\partial \Omega$ is a locally finite measure. Also, the function $u$ is holomorphic in $\Omega$, and the conditions in (3.6) are satisfied for any $\kappa>0$. In addition, $\left(\left.u\right|_{\partial \Omega} ^{\text {n.t. }}\right)(\zeta)=\frac{1}{\zeta}$ for every $\zeta \in \partial B(0,1)$. If we now fix a point $z \in \Omega$ and define $f(\zeta):=\frac{1}{\zeta(\zeta-z)}$ for $\zeta \in \mathbb{C} \backslash\{z, 0\}$, we have that $f$ is meromorphic with poles of order one at 0 and $z$. Hence, the boundary integral in the right-hand side of (3.8) may be computed using the Residue Theorem as

$$
\begin{aligned}
\int_{\partial B(0,1)} f(\zeta) d \zeta & =2 \pi i[\operatorname{Res}(f, z)+\operatorname{Res}(f, 0)] \\
& =2 \pi i\left[\frac{1}{z}-\frac{1}{z}\right]=0 .
\end{aligned}
$$

Given that the solid integral in (3.8) is zero, if (3.8) were 
to hold in this case, we would obtain $u(z)=0$ for each $z \in \Omega$, a contradiction.

We also wish to stress that both assumptions in (3.6) are necessary. To see that this is the case, consider the slit disk $\Omega:=\{z \in B(0,1): z \notin[0,1)\}$ and bring in the holomorphic function $u: \Omega \rightarrow \mathbb{C}$ given by $u(\zeta):=\frac{1}{\zeta}$ for each $\zeta \in \Omega$. Then the boundary integral in (3.8) is just as in (3.22), hence zero. Thus, (3.8) becomes $u(z)=0$ for each $z \in \Omega$, a contradiction. In this scenario, $\Omega$ satisfies all geometric hypotheses stipulated in Theorem 3.2, and $u$ satisfies all but the first condition in (3.6). The latter presently fails. Specifically, since for each fixed $\kappa>0$ we have $\left(\mathcal{N}_{\kappa} u\right)(x) \approx x^{-1}$ uniformly for $x \in(0,1) \subseteq \partial \Omega$, it follows that

$$
\mathcal{N}_{\kappa} u \in L^{1, \infty}(\partial \Omega, \sigma) \text { but } \mathcal{N}_{\kappa} u \notin L^{1}(\partial \Omega, \sigma) .
$$

This shows that the first condition in (3.6) is indeed necessary.

As regards the necessity of the second condition in (3.6), consider the open subset of $\mathbb{C}$ described as

$$
\Omega:=\left\{z \in B(0,1): z \notin\left[-\frac{1}{2},+\frac{1}{2}\right] \times\{0\}\right\},
$$

pick some $f \in \mathcal{C}_{c}^{0}\left(\left(-\frac{1}{2},+\frac{1}{2}\right)\right)$ which is not identically zero, then define the function

$$
u(z):=\frac{1}{2 \pi i} \int_{-1 / 2}^{1 / 2} \frac{f(t)}{z-t} d t \quad \forall z \in \Omega .
$$

Then $u$ is holomorphic in $\Omega$, and

$$
\left(\left.u\right|_{\partial \Omega} ^{\text {n.t. }}\right)(\zeta)=\frac{1}{2 \pi i} \int_{-1 / 2}^{1 / 2} \frac{f(t)}{\zeta-t} d t
$$$$
\text { for each } \zeta \in \partial_{*} \Omega=\partial B(0,1) \text {. }
$$

Consequently, Fubini's Theorem implies that for each point $z \in \Omega$ we have

$$
\begin{aligned}
\int_{\partial_{*} \Omega} & \frac{\left(\left.u\right|_{\partial \Omega} ^{\text {n.t. }}\right)(\zeta)}{\zeta-z} d \zeta \\
\quad= & \frac{1}{2 \pi i} \int_{\partial B(0,1)}\left(\int_{-1 / 2}^{1 / 2} \frac{f(t)}{\zeta-t} d t\right) \frac{d \zeta}{\zeta-z} \\
\quad= & \int_{-1 / 2}^{1 / 2} f(t)\left(\frac{1}{2 \pi i} \int_{\partial B(0,1)} \frac{d \zeta}{(\zeta-t)(\zeta-z)}\right) d t \\
& =0,
\end{aligned}
$$

since by the Residue Theorem the last integral on the unit circle vanishes. As such, if the integral representation formula (3.8) were to hold, it would presently imply that $u(z)=0$ for each $z \in \Omega$. However, this is not the case.
For example, for $\mathcal{L}^{1}$-a.e. $x \in\left(-\frac{1}{2},+\frac{1}{2}\right)$ we have

$$
\begin{aligned}
\lim _{y \rightarrow 0^{+}} u(x \pm i y)= & \pm \frac{1}{2} f(x) \\
& +\lim _{\varepsilon \rightarrow 0^{+}} \frac{1}{2 \pi i}\left(\int_{-1 / 2}^{x-\varepsilon}+\int_{x+\varepsilon}^{+1 / 2}\right) \frac{f(t)}{z-t} d t,
\end{aligned}
$$

implying that

there exists some Lebesgue measurable set $A \subseteq\left(-\frac{1}{2},+\frac{1}{2}\right)$ with $\mathcal{L}^{1}(A)>0$ such that

$\lim _{y \rightarrow 0^{+}} u(x+i y)-\lim _{y \rightarrow 0^{+}} u(x-i y)=f(x) \neq 0$

for each point $x \in A$.

In the current setting, $\Omega$ satisfies all geometric hypotheses stipulated in Theorem 3.2, and $u$ satisfies all but the second condition in (3.6). However, (3.27) proves that the latter fails and, even though $\left.u\right|_{\partial \Omega} ^{k \text {-n.t. }}$ does exist at $\sigma$-a.e. point on $\partial_{*} \Omega$, the failure of the second condition in (3.6) ultimately invalidates (3.8).

As the reader surely suspects by now, Theorems 2.5-2.6 have many other fundamental applications, such as sharp versions of all Green integral identities for second-order operators (including boundary layer potential representations for solutions of second-order elliptic PDE's) in $\mathbb{R}^{n}$.

We, however, shall stay in the complex plane and conclude with a very general version of the classical Residue Theorem in complex analysis.

Theorem 3.3. Let $\Omega \subseteq \mathbb{R}^{2} \equiv \mathbb{C}$ be an open set with $a$ lower Ahlfors regular boundary, and with the property that $\sigma:=\mathcal{H}^{1}\lfloor\partial \Omega$ is a doubling measure on $\partial \Omega$. Suppose $f$ is a meromorphic function in $\Omega$ whose poles are contained in some compact set $K \subset \Omega$. For some aperture parameter $\kappa>0$, assume that

$$
\begin{aligned}
& \mathcal{N}_{\mathcal{K}}^{\Omega \backslash K}\left(\left.f\right|_{\Omega \backslash K}\right) \in L^{1}(\partial \Omega, \sigma) \text { and } \\
& \left.f\right|_{\partial \Omega} ^{\kappa-n . t .} \text { exists }(\text { in } \mathbb{C}) \text { at } \sigma \text {-a.e. point on } \partial_{\mathrm{nta}} \Omega \text {. }
\end{aligned}
$$

Then for any $\kappa^{\prime}>0$ the nontangential trace $\left.f\right|_{\partial \Omega} ^{\kappa^{\prime}-\text { n.t. }}$ exists at $\sigma$-a.e. point on $\partial_{n t a} \Omega$ and is actually independent of $\kappa^{\prime}$. When regarding the latter function as being defined $\sigma$-a.e. on $\partial_{*} \Omega$, this belongs to $L^{1}\left(\partial_{*} \Omega, \sigma\right)$ and, with the dependence on the parameter $\mathcal{K}^{\prime}$ dropped,

$$
2 \pi i \cdot \sum_{z \text { pole of } f} \operatorname{Res}(f, z)=\int_{\partial_{*} \Omega}\left(\left.f\right|_{\partial \Omega} ^{\text {n.t. }}\right)(\zeta) d \zeta
$$

in the case when either $\Omega$ is bounded, or $\partial \Omega$ is unbounded. Finally, when $\Omega$ is an exterior domain, formula (3.29) continues to hold under the additional assumption that there exists some $\lambda \in(1, \infty)$ such that $\int_{B(0, \lambda R) \backslash B(0, R)}|f| d \mathcal{L}^{2}=o(R)$ as $R \rightarrow \infty$. 
Proof. Let $\left\{z_{j}\right\}_{1 \leq j \leq N} \subseteq K$ be the poles of the meromorphic function $f$. More specifically, assume that there exists a family $\left\{\mathcal{O}_{j}\right\}_{1 \leq j \leq N}$ of mutually disjoint open subsets of $\Omega$ with the property that for each $j \in\{1, \ldots, N\}$ we have $z_{j} \in$ $\mathcal{O}_{j}$ and we may find a holomorphic function $g_{j}: \mathcal{O}_{j} \rightarrow \mathbb{C}$ with $g_{j}\left(z_{j}\right) \neq 0$ along with an integer $m_{j} \in \mathbb{N}$ such that $f(z)=g_{j}(z) /\left(z-z_{j}\right)^{m_{j}}$ for each point $z \in \mathcal{O}_{j} \backslash\left\{z_{j}\right\}$. Then for each $j \in\{1, \ldots, N\}$ it follows that $z_{j}$ is a pole of order $m_{j}$ for $f$ and

$$
\begin{aligned}
\operatorname{Res}\left(f, z_{j}\right) & =\frac{1}{\left(m_{j}-1\right) !} \lim _{z \rightarrow z_{j}}\left(\frac{\partial}{\partial z}\right)^{m_{j}-1}\left[\left(z-z_{j}\right)^{m_{j}} f(z)\right] \\
& =\frac{1}{\left(m_{j}-1\right) !} g_{j}^{\left(m_{j}-1\right)}\left(z_{j}\right) .
\end{aligned}
$$

Next, extend $f \in \mathcal{C}^{\infty}\left(\Omega \backslash\left\{z_{1}, \ldots, z_{N}\right\}\right)$ to a distribution $u$ in $\Omega$ by taking $u$ to be in each $\mathcal{O}_{j}$ the distribution

$$
\frac{(-1)^{m_{j}-1}}{\left(m_{j}-1\right) !} g_{j}\left(\frac{\partial}{\partial z}\right)^{m_{j}-1}\left[\frac{1}{z-z_{j}}\right]
$$

where the expression in brackets is regarded as a locally integrable function and the subsequent iterated derivatives in $z$ are taken in the sense of distributions. Hence

$$
u \in \mathcal{D}^{\prime}(\Omega) \text { and }\left.u\right|_{\Omega \backslash\left\{z_{1}, \ldots, z_{N}\right\}}=\left.f\right|_{\Omega \backslash\left\{z_{1}, \ldots, z_{N}\right\}} .
$$

Let us also consider the distribution $w \in \mathcal{E}^{\prime}(\Omega)$ given by

$$
\sum_{j=1}^{N} \sum_{k=0}^{m_{j}-1} \frac{\pi(-1)^{k}}{\left(m_{j}-1\right) !}\left(\begin{array}{c}
m_{j}-1 \\
k
\end{array}\right) g_{j}^{\left(m_{j}-1-k\right)}\left(z_{j}\right)\left(\frac{\partial}{\partial z}\right)^{k} \delta_{z_{j}} .
$$

Since $\bar{\partial}\left[1 /\left(z-z_{j}\right)\right]=\pi \delta_{z_{j^{\prime}}}$ one may easily check that $\bar{\partial} u$ coincides with $w$ in each $\mathcal{O}_{j}$ with $1 \leq j \leq N$ (see, e.g., [16, Theorem 7.43, p. 289] and [16, Exercise 2.45, p. 34]). Given that we also know that both $\bar{\partial} u$ and $w$ vanish in the set $\Omega \backslash\left\{z_{1}, \ldots, z_{N}\right\}$, we ultimately conclude that

$$
w=\bar{\partial} u \text { in } \mathcal{D}^{\prime}(\Omega)
$$

As seen from (3.30) and the definition of $w$, the pairing $\mathcal{E}^{\prime}(\Omega)\langle w, 1\rangle_{\mathcal{E}(\Omega)}$ equals

$$
\sum_{j=1}^{N} \frac{\pi}{\left(m_{j}-1\right) !} g_{j}^{\left(m_{j}-1\right)}\left(z_{j}\right)=\pi \cdot \sum_{z \text { pole of } f} \operatorname{Res}(f, z) .
$$

In concert with (3.33), this implies that

$$
\begin{aligned}
\left(\mathcal{C}_{b}^{\infty}(\Omega)\right)^{*}(\bar{\partial} u, 1)_{\mathcal{C}_{b}^{\infty}(\Omega)} & ={ }_{\mathcal{E}^{\prime}(\Omega)}\langle\bar{\partial} u, 1\rangle_{\mathcal{E}(\Omega)} \\
& =\pi \cdot \sum_{z \text { pole of } f} \operatorname{Res}(f, z) .
\end{aligned}
$$

If we now consider the vector field

$$
\vec{F}:=(u, i u) \in\left[\mathcal{D}^{\prime}(\Omega)\right]^{2},
$$

then from (3.28) and (3.32) we conclude that

$$
\begin{gathered}
\left.\left.\vec{F}\right|_{\partial \Omega} ^{\kappa-\text { n.t. }} \quad \text { exists (in } \mathbb{C}^{2}\right) \sigma \text {-a.e. on } \partial_{\text {nta }} \Omega, \\
\left.\vec{F}\right|_{\Omega \backslash K} \in\left[L_{\text {loc }}^{1}\left(\Omega \backslash K, \mathcal{L}^{2}\right)\right]^{2} \text {, and } \\
\mathcal{N}_{\mathcal{K}}^{\Omega \backslash K}\left(\left.\vec{F}\right|_{\Omega \backslash K}\right) \in L^{1}(\partial \Omega, \sigma) .
\end{gathered}
$$

In addition,

$$
\operatorname{div} \vec{F}=\partial_{x} u+i \partial_{y} u=2 \bar{\partial} u \text { in } \mathcal{D}^{\prime}(\Omega) ;
$$

hence $\operatorname{div} \vec{F} \in \mathcal{E}^{\prime}(\Omega)$. Moreover, in the case when $\Omega$ is an exterior domain, the decay of $f$ implies that $\vec{F}$ satisfies (2.19). Finally, at $\sigma$-a.e. point on $\partial_{*} \Omega$ we have

$$
\begin{aligned}
\left\langle\nu,\left.\vec{F}\right|_{\partial \Omega} ^{\kappa-\text { n.t. }}\right\rangle & =v_{1}\left(\left.f\right|_{\partial \Omega} ^{\kappa-\text { n.t. }}\right)+i v_{2}\left(\left.f\right|_{\partial \Omega} ^{\kappa-\text { n.t. }}\right) \\
& =v\left(\left.f\right|_{\partial \Omega} ^{\kappa-\text { n.t. }}\right) .
\end{aligned}
$$

At this stage, (3.29) follows from Theorem 2.6, bearing in mind (3.5), (3.35), (3.34), and (3.36).

This version of the Residue Theorem is actually more efficient than the standard technology (based on choosing a suitable contour of integration, evaluating various integrals, and passing to the limit) even in such mundane scenarios as the task of showing that

$$
\int_{-\infty}^{+\infty} \frac{e^{i x}}{x^{2}+1} d x=\frac{\pi}{e}
$$

Specifically, choosing $\Omega:=\mathbb{R}_{+}^{2}$ and considering the meromorphic function

$$
f(z):=\frac{e^{i z}}{z^{2}+1} \text { for } z \in \Omega,
$$

$f$ has a simple pole at $z=i$, with residue $e^{-1}$, the function $f$ may be extended continuously to a neighborhood of $\partial \Omega$, and $\int_{\partial \Omega}|f| d \sigma=\int_{\mathbb{R}} \frac{d x}{x^{2}+1}<+\infty$.

Incidentally, it is not much harder to see that the first condition in (3.28) is true in this case. Indeed, if we set $K:=\overline{B(i, 1 / 2)}$, then for each $\kappa>0$ fixed we deduce (keeping in mind that $\left|e^{i z}\right| \leq 1$ for each $z \in \Omega$ ) that

$$
\begin{gathered}
\left(\mathcal{N}_{\mathcal{K}}^{\Omega \backslash K}\left(\left.f\right|_{\Omega \backslash K}\right)\right)(x) \approx \frac{1}{x^{2}+1}, \\
\text { uniformly for } x \in \mathbb{R} \equiv \partial \Omega,
\end{gathered}
$$

from which the desired conclusion follows. Thus, (3.29) holds and this gives (3.37).

ACKNOWLEDGMENTS. The authors are grateful to the referee for a careful reading. The authors were supported in part by the Simons Foundation grants 426669, 616050, and 637481; a Simons Fellowship at the Isaac Newton Institute; and the NSF DMS grant 1900938. 


\section{References}

[1] A. Cauchy, Sur les intégrales qui s'étendent à tous les points d'une courbe fermée, Comptes Rendus 23 (1846), 251-255.

[2] Gui-Qiang Chen, Monica Torres, and William P. Ziemer, Gauss-Green theorem for weakly differentiable vector fields, sets of finite perimeter, and balance laws, Comm. Pure Appl. Math. 62 (2009), no. 2, 242-304, DOI 10.1002/cpa.20262. MR2468610

[3] Björn E. J. Dahlberg and Carlos E. Kenig, Hardy spaces and the Neumann problem in $L^{p}$ for Laplace's equation in Lipschitz domains, Ann. of Math. (2) 125 (1987), no. 3, 437-465, DOI 10.2307/1971407. MR890159

[4] Ennio De Giorgi, Complementi alla teoria della misura $(n-1)$-dimensionale in uno spazio $n$-dimensionale (Italian), Seminario di Matematica della Scuola Normale Superiore di Pisa, 1960-61, Editrice Tecnico Scientifica, Pisa, 1961. MR0179650

[5] Ennio De Giorgi, Frontiere orientate di misura minima (Italian), Seminario di Matematica della Scuola Normale Superiore di Pisa, 1960-61, Editrice Tecnico Scientifica, Pisa, 1961. MR0179651

[6] Lawrence C. Evans and Ronald F. Gariepy, Measure theory and fine properties of functions, Studies in Advanced Mathematics, CRC Press, Boca Raton, FL, 1992. MR1158660

[7] Herbert Federer, The Gauss-Green theorem, Trans. Amer. Math. Soc. 58 (1945), 44-76, DOI 10.2307/1990234. MR13786

[8] Herbert Federer, A note on the Gauss-Green theorem, Proc. Amer. Math. Soc. 9 (1958), 447-451, DOI 10.2307/2033002 MR95245

[9] C. F. Gauss, Theoria attractionis corporum sphaeroidicorum ellipticorum homogeneorum methodo nova tractata, Commentationes societatis regiae scientiarium Gottingensis recentiores 2 (1813), 355-378.

[10] G. Green, An Essay on the Application of Mathematical Analysis to the Theories of Electricity and Magnetism, T. Wheelhouse, Nottingham, England, 1828.

[11] Steve Hofmann, Marius Mitrea, and Michael Taylor, Singular integrals and elliptic boundary problems on regular Semmes-Kenig-Toro domains, Int. Math. Res. Not. IMRN 14 (2010), 2567-2865, DOI 10.1093/imrn/rnp214. MR2669659

[12] Victor J. Katz, The history of Stokes' theorem, Math. Mag. 52 (1979), no. 3, 146-156, DOI 10.2307/2690275. MR533433

[13] Carlos E. Kenig and Tatiana Toro, Poisson kernel characterization of Reifenberg flat chord arc domains (English, with English and French summaries), Ann. Sci. École Norm. Sup. (4) 36 (2003), no. 3, 323-401, DOI 10.1016/S00129593(03)00012-0. MR1977823

[14] Joseph Louis Lagrange, Oeuvres. Tome 1 (French), Georg Olms Verlag, Hildesheim-New York, 1973. Publiées par les soins de J.-A. Serret; Avec une notice sur la vie et les ouvrages de J.-L. Lagrange par J.-B. J. Delambre; Nachdruck der Ausgabe Paris 1867. MR0439546

[15] José María Martell, Dorina Mitrea, Irina Mitrea, and Marius Mitrea, The BMO-Dirichlet problem for elliptic systems in the upper half-space and quantitative characterizations of VMO, Anal. PDE 12 (2019), no. 3, 605-720, DOI 10.2140/apde.2019.12.605. MR3864207

[16] Dorina Mitrea, Distributions, Partial Differential Equations, and Harmonic Analysis, Universitext, Springer, Cham, 2018. Second edition [ MR3114783]. MR3887685

[17] D. Mitrea, I. Mitrea, and M. Mitrea, A Sharp Divergence Theorem with Non-Tangential Pointwise Traces and Applications to Singular Integrals and Boundary Problems, book manuscript, 2019.

[18] Dorina Mitrea, Irina Mitrea, Marius Mitrea, and Michael Taylor, The Hodge-Laplacian: Boundary value problems on Riemannian manifolds, De Gruyter Studies in Mathematics, vol. 64, De Gruyter, Berlin, 2016. MR3586566

[19] Irina Mitrea, Marius Mitrea, and Michael Taylor, Cauchy integrals, Calderón projectors, and Toeplitz operators on uniformly rectifiable domains, Adv. Math. 268 (2015), 666-757, DOI 10.1016/i.aim.2014.09.020 MR3276607

[20] M. V. Ostrogradsky, Démonstration d'un théorème du calcul intégral, communicated to the Paris Academy on February 13, 1826.

[21] B. Riemann, Grundlagen für eine allgemeine Theorie der Functionen einer veränderlichen complexen Grösse, Göttingen, 1867.

[22] Charles H. Stolze, A history of the divergence theorem (English, with French summary), Historia Math. 5 (1978), no. 4, 437-442, DOI 10.1016/0315-0860(78)90212-4 MR511180

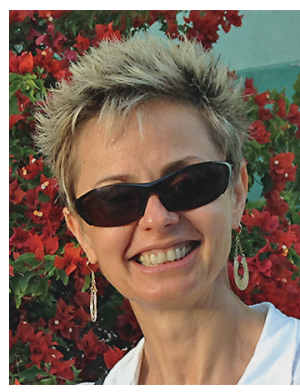

Dorina Mitrea

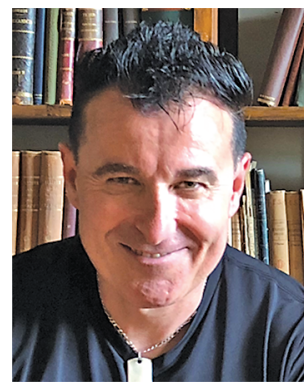

Marius Mitrea

Credits

Opening graphic is courtesy of MaryliaDesign via Getty. Author photos are courtesy of the authors. 